\title{
Development and Application of Imaging in Therapeutic Studies
}

National Cancer Institute

\section{Source}

National Cancer Institute. Development and Application of Imaging in Therapeutic

Studies. NCI Thesaurus. Code C15946.

This initiative will encourage applications that apply imaging technologies in the assessment of investigational cancer therapeutic agents. The proposed research projects will focus on the development and application of non-invasive tools for the functional or quantitative assessment of biochemical, genetic, or pharmacologic activity of specific therapeutic agents, or the defined biochemical pathway(s) in which they act. (from an RFA description) 